\title{
Process Dissociation, Cognitive Architecture, and Response Time: Comments on Lindsay and Jacoby (1994)
}

\author{
Anne P. Hillstrom and Gordon D. Logan \\ University of Illinois at Urbana-Champaign
}

\begin{abstract}
Process dissociation is based on 2 assumptions about the processes being dissociated: invariance of the processes across situations, and stochastic independence of the processes. In a recent application of process dissociation to the Stroop task (D. S. Lindsay \& L. L. Jacoby, 1994), both of those assumptions were violated. It is argued that these violations were due to (a) an oversimplification of the processing architecture that ignores common stages such as guessing and response selection, (b) an assumption that the more automatic process (word reading) dominates over the intended process (color naming) in determining responses, and (c) an assumption that switching from the more common speeded response instruction (measuring speed) to a deadline response instruction (measuring accuracy) does not change processing. General implications for applying process dissociation to dynamic tasks are discussed.
\end{abstract}

Process dissociation is a method for determining how task accuracy is influenced by cognitive processes that are capable of either competing or working in concert (Jacoby, 1991). It has been used primarily to show relative contributions of implicit and explicit memory in different tasks, but it has the potential to be used for other issues as well. Recently, Lindsay and Jacoby (1994) extended process dissociation to a visual identification task, the Stroop task, which appears to have underlying processes that sometimes compete and sometimes work in concert. The process dissociation method puts few explicit constraints on processing. But when we considered in detail how processing would be constrained by the assumptions of the method, the results are problematic, partly in ways addressed by earlier criticisms and partly in ways unique to the new domain. In particular, when one tries to reconcile this work with earlier work on understanding processing architecture of the Stroop task, three issues appear problematic: the assumption of processing independence, the side effects of applying deadlines to a dynamic process, and the characterization of automatic processing.

Anne P. Hillstrom and Gordon D. Logan, Department of Psychology, University of Illinois at Urbana-Champaign.

This work was supported by National Institute of Mental Health National Research Service Award Fellowship MH11064-02 and National Science Foundation Grant SBR 94-10406. We thank Steve Lindsay and Larry Jacoby for helpful conversations about the ideas expressed; Dan Spieler and Tim Curran for helpful comments on the article; and Julie Delheimer for testing subjects.

Correspondence concerning this article should be addressed to Anne P. Hillstrom, who is now at School of Psychology, University of Wales, Bangor, Gwynedd, Wales, United Kingdom, LL57 2DG, or to Gordon D. Logan, Department of Psychology, University of Illinois at Urbana-Champaign, 603 East Daniel, Champaign, IL 61820. Electronic mail may be sent via Internet to ap. hillstrom @bangor. ac.uk or to glogan@s.psych.uiuc.edu, respectively.

\section{Process Dissociation}

Process dissociation is a method of solving simultaneous equations that represent how internal processes work in conjunction to accomplish tasks. The equations represent a framework of a model of processing. Although much more detail would be needed to account for all aspects of responding to a task, only a minimal framework of a model is required to use the processing dissociation method. The equations represent different ways that processes work together in different situations. The algebraic requirement for finding the influence of all $n$ internal processes represented in the equations is that there must be $n$ independent equations reflecting different relationships between the variables.

The original applications of process dissociation logic tested a two-factor theory of memory, using two equations to solve for the influence of each factor on two kinds of responses. Because the Stroop application also proposes a two-process theory, we focus on a two-process case example in our description of the method (see Yonelinas \& Jacoby, 1995, for an example using four equations and four unknowns). In a memory study that is typical of the way process dissociation is used, Jacoby, Toth, and Yonelinas (1993) had subjects study a set of words and then perform stem completion. Subjects were asked either (a) to try to complete the stem with words from the study set (the inclusion condition) or (b) to try to complete the stem with words not from the study set (the exclusion condition). The equations modeling processing in the two conditions were

$p$ (studied word, inclusion condition)

and

$$
=p(R)+p(A)(1-p(R)),
$$

$p($ studied word, exclusion condition $)=p(A)(1-p(R))$,

where $p(R)$ was the probability of responding with a studied 
word based on recognition, or explicit memory, and $p(A)$ was the probability of responding with a studied word based on automatic processes, or implicit memory. In other words, subjects in the inclusion condition responded with the word either because they remembered studying it or because implicit factors brought it to mind, whereas subjects in the exclusion condition responded with the word only when they did not remember studying it but implicit factors brought it to mind anyway. This characterization of the exclusion condition assumes that explicit memory dominates over implicit memory in the sense that the effect of implicit memory is seen only when explicit memory fails.

The probability of completing the stem with a studied word was estimated empirically under the inclusion and exclusion instructions, and the two variables were solved for. Note that for the solution to be meaningful, two assumptions were required: The way in which explicit and implicit memory work must be the same regardless of instruction type (invariance, which allowed the equations to be combined mathematically when solving for the unknowns), and the two processes being dissociated must be stochastically independent (independence). ${ }^{1}$

The equations used embody the independence assumption: When $R$ and $A$ are independent, then $p(R$ or $A)=$ $p(R)+p(A)(1-p(R))$. These equations are valid if and only if $p(R)$ and $p(A)$ are stochastically independent. If the independence assumption is wrong, solutions for $p(R)$ and $p(A)$ under the current equations will be wrong (Curran \& Hintzmann, 1995, 1997; Jacoby, Begg, \& Toth, 1997).

In general, solving the two equations informs about the relative influence of Process 1 and Process 2 under the specific context conditions in which the experiment was run. Process dissociation is typically embedded in two or more sets of context conditions to test how changes in context affect the relationship between Process 1 and Process 2. For instance, Jacoby et al. (1993) dissociated retrieval processes after subjects either fully attended or divided their attention between tasks during study of the test words, and showed that their attention manipulation affected the influence of explicit but not implicit memory.

\section{The Stroop Task}

The Stroop task is one in which subjects name the color in which words appear, where the words themselves are usually names of colors (Stroop, 1935). Research on this task has shown robust congruency effects: Subjects respond faster when the word is a color name that matches the color of the word than when the word is a color name different from the color of the word (MacLeod, 1991). Response times for neutral stimuli (e.g., a color patch, a nonword letter string, or a word unrelated to colors) are typically between response times for congruent and incongruent stimuli. Accuracy typically is very high in these experiments. Subjects can easily limit errors to fewer than $10 \%$ of responses. Thus, in these experiments, which do not typically impose response deadlines, the Stroop effect is predominantly a response time effect; effects of congruency on accuracy have been small and often not significant.
Models of processing underlying the Stroop effect have been held accountable for explaining both parts of the effect of incongruence between word and color: that processing is slowed, and that people are able to perform accurately. Although no one theory of Stroop processing predominates, most theories have assumed that the difficulty of the Stroop task is due to the differences in speed and automaticity of the two cognitive processes. Reading a word is a fast, automatic process; naming a color is a less automatic process that is invoked deliberately and that proceeds more slowly. When the word is the name of a color different from the color to be named, a conflicting response (the word) is generally available easily and faster than the correct response. Models of the Stroop task have often invoked a response resolution stage to explain why emitted responses are nonetheless correct, and the effect of congruency on the duration of this stage accounts for the response time effect (e.g., Cohen, Dunbar, \& McClelland, 1990; Logan, 1980; Phaf, van der Heijden, \& Hudson, 1990; also see Eriksen \& Schultz, 1979).

\section{Dissociation of the Stroop Task}

Because models of the Stroop effect assume that the differences between responses to congruent stimuli and responses to incongruent stimuli lie in the coordination of the output of the word reading and color naming processes, the Stroop effect seems an ideal candidate for the application of the process dissociation method. Lindsay and Jacoby (1994) did just that. This represents a major extension of process dissociation; for the first time the method was applied to a cognitive task that has traditionally been measured in terms of speed, not accuracy.

Applying process dissociation required measuring the Stroop effect in terms of accuracy rather than speed. In order to have a measurable accuracy effect, Lindsay and Jacoby (1994) imposed deadlines for responses. They believed that doing so would shift the Stroop effect from speed to accuracy with little effect on underlying processing. This could happen if subjects given a deadline processed stimuli in the same manner but shifted their speed-accuracy criterion to generate responses of faster and less variable speed, but of lower and more variable accuracy.

Lindsay and Jacoby (1994) used the following equations to describe the contribution of color naming and word reading in the congruent stimuli and incongruent stimuli conditions:

$$
p(\text { correct } \mid \text { congruent })=W+C(1-W)
$$

and

$$
p(\text { correct } \mid \text { incongruent })=C(1-W)
$$

\footnotetext{
${ }^{1}$ Note that the assumption of stochastic independence, which is usually taken to be an assumption of the method, is instead an assumption of all models to which the method has so far been applied. See Joordens and Merikle (1993) for a discussion of how process dissociation could be applied to a model that does not assume process independence.
} 
where $W$ is the probability of word reading controlling the response and $C$ is the probability of color naming controlling the response. Word reading and color naming were assumed to be stochastically independent and to work the same way in congruent and incongruent conditions (i.e., to be invariant).

By solving Equations 3 and 4, Lindsay and Jacoby (1994) estimated the contribution of color naming and word reading to responses. Lindsay and Jacoby evaluated the reasonableness of their model in two ways. They showed that the $p$ (correct|neutral) was correlated to some degree with $C$ ( $r=.69$ for the $800 \mathrm{~ms}$ post hoc deadline, $r=.74$ for the $600 \mathrm{~ms}$ post hoc deadline), which was a prediction of the model. And they demonstrated functional independence between word reading and color naming by showing that estimates of $W$ but not $C$ were affected by changes to the proportion of congruent, incongruent, and neutral trials, whereas estimates of $C$ but not $W$ were affected by changes to color quality.

\section{Concerns About Characterization of Processing}

Lindsay and Jacoby (1994) feel that the strength of their work lies in (a) the introduction of a method for estimating the contribution of color naming and word reading to Stroop task performance and (b) the conclusion that facilitation and interference cannot be measured by comparing performance to a baseline. The focus of our work is to evaluate the method introduced, but before doing that we feel that the second conclusion deserves comment. As Lindsay and Jacoby noted, Jonides and Mack (1984) already pointed out that measuring facilitation and inhibition against a neutral baseline in response time domains is undesirable. For that reason, despite a fair amount of research searching for an appropriate baseline, researchers have not settled on one. As a result, many researchers (e.g., Brown, Roos-Gilbert, \& Carr, 1995; Kahneman \& Chajczyk, 1983; Logan \& Zbrodoff, 1979) have compared performance for congruent stimuli with performance for incongruent stimuli rather than comparing performance for both with performance for neutral stimuli, just as Lindsay and Jacoby did.

That said, we believe this application of process dissociation is problematic. Most of our concerns are about the way processing is characterized. Specifically, we question (a) the assumed stochastic independence of word reading and color naming, (b) whether imposing deadlines on the Stroop task affects processing as indicated, and (c) whether the automaticity of word reading is represented correctly in the equations.

In order to explain our concerns, we must first explain possible interpretations of the framework provided by the process dissociation equations, and what implications they have for underlying architecture. Then we address the general issues as they apply to those frameworks.

\section{What Are $W$ and $C$ ?}

Consider what $W$ and $C$ mean in Equations 3 and 4. Lindsay and Jacoby (1994) alternately described $W$ and $C$ as probabilities that one or the other process "influences" or "controls" overt responses (p. 220). $W$ and $C$ reflect re-

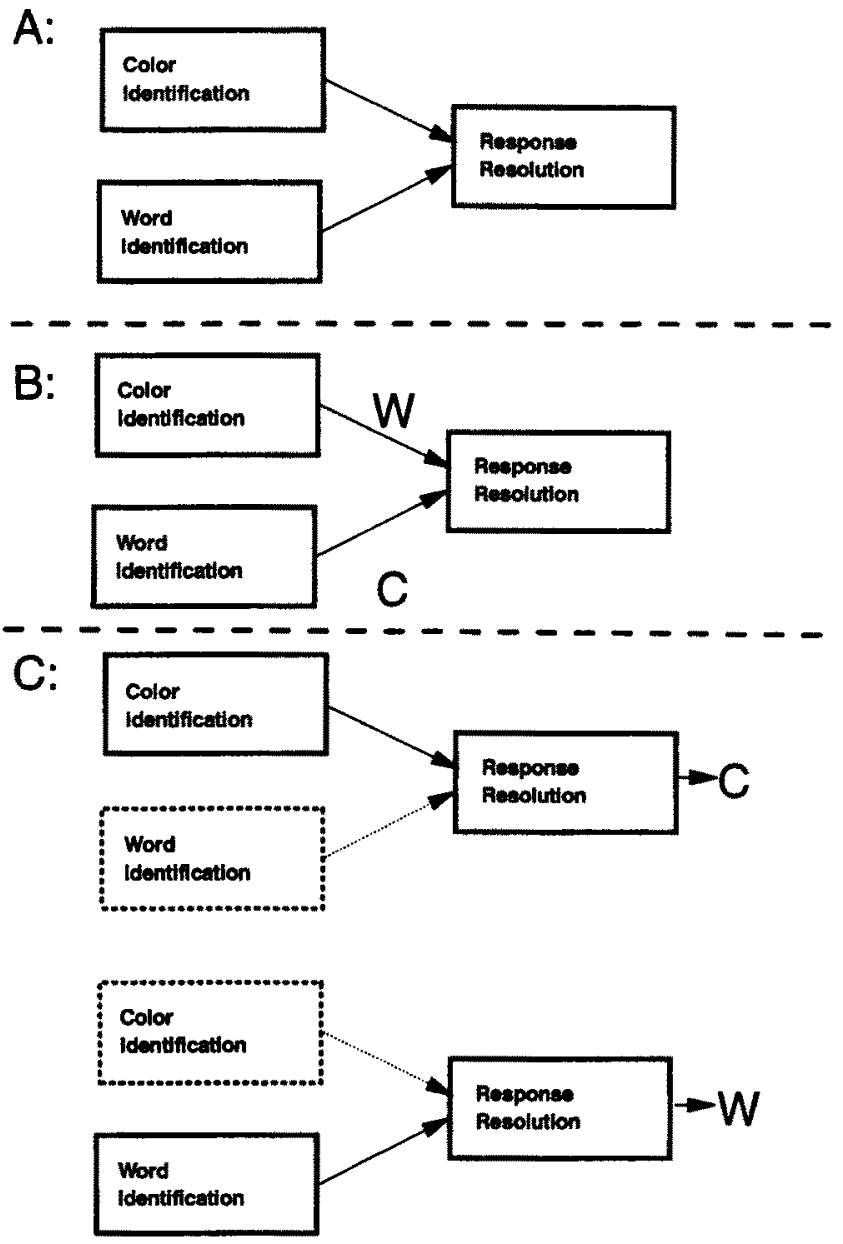

Figure 1. A: A standard box model of the Stroop task. B: A box model showing $W$ and $C$ interpreted as component reliabilities. $\mathrm{C}$ : A box model showing $W$ and $C$ interpreted as probabilities of responses being derived from one path or another through the system.

sponse probabilities: If word identification completes successfully but does not affect the response made, it will not be reflected in $W$. However, there is ambiguity about what the words influence and control mean in this context. Because there seems to be some confusion about what $W$ and $C$ mean, we now outline three possible interpretations in detail.

$W$ and $C$ as component or path reliabilities. At a high level, all standard models of the Stroop task can be represented as in Panel A of Figure 1, where word identification and color identification feed into a common response resolution stage. One way to understand $W$ and $C$ is to consider them as the probabilities that word identification and color identification, respectively, complete successfully. Accordingly, $W$ and $C$ are component reliabilities (see Panel B of Figure 1). ${ }^{2}$ Inherent in such a characterization is the

\footnotetext{
2 We are using the engineering definition of reliability here: The reliability of a component or circuit is the probability that it will not fail to perform its function.
} 
assumption that response resolution is accomplished by rules that always work successfully. The probability of producing a correct response is not affected by the probability of a response resolution process completing in a certain way.

Responses are determined as follows. If only one component finishes, its output is chosen as the response. Inherent in Equations 3 and 4 is the response resolution rule for when the word and the color are both identified: The word is chosen as the output. Only when the word identification process fails to produce a response will the output of the color identification process be chosen as output.

This interpretation is consistent with Equations 3 and 4, which instantiate both the response resolution rule (dominance of word reading over color naming) and the independence assumption. However, this interpretation is inconsistent with the data resulting from process dissociation. Lindsay and Jacoby's (1994) results show that $W$ decreases over time (see their Figure 3 for an example). Because of the dominance assumption, $W$ closely reflects the probability that the word identification completes successfully. It is illogical to believe that a process will be less likely to complete successfully when given more time to finish. In other words, the probability that a process finishes within 600 ms must be larger than (or at least not smaller than) the probability that the same process finishes within $400 \mathrm{~ms}$.

$W$ and $C$ as path reliabilities. According to an alternative interpretation, $W$ and $C$ represent not the reliability of individual components, but the probabilities that the response was derived by taking one or the other path through the system. As shown in Panel C of Figure $1, W$ is the probability that the word was identified and the response resolution process chose it as the output. $C$ is the probability that the color was identified and the response resolution process chose it as the output.

The biggest problem with this interpretation is that $W$ and $C$ are not independent probabilities. They are probabilities of mutually exclusive occurrences. Equations 3 and 4, which reflect independence of $W$ and $C$, would not be valid. Equations like the following would better represent this interpretation:

$$
p(\text { correct } / \text { congruent })=C+W,
$$

and

$$
p \text { (correct } \mid \text { incongruent })=C \text {. }
$$

The results can no longer be evaluated in terms of predictions about $p$ (correct|neutral), because the neutral condition would not be affected by response selection. But the results could be evaluated by the following prediction:

$$
p(\text { word } \mid \text { incongruent })=W \text {. }
$$

This interpretation abandons the assumed independence of word reading and color naming and does not require an explicit assumption of word reading dominating over color naming, so it does not seem to be the interpretation intended by Lindsay and Jacoby (1994). Because this interpretation is consistent with some of the text, however, some readers may have had it in mind.

$W$ and $C$ as influences. According to the above two interpretations of $W$ and $C$, the response made is generated wholly from one or the other process, not from output combined from both processes. An individual response cannot be influenced by both the word reading process and the color naming process. This interpretation seems consistent with the following quote: "there are two ways a correct response can be produced on a congruent item: either because word-reading processes control the response or because color-naming processes control the response" (Lindsay \& Jacoby, 1994, p. 220). This feature of the above interpretations of $W$ and $C$ is consistent with models like race models or deadline models and is inconsistent with models like counter or random walk models, in which the results of word identification and color identification are integrated to form the response. The most successful models of the Stroop task to date are the latter type (e.g., Cohen et al., 1990; Logan, 1980; Logan \& Zbrodoff, 1979).

Because Lindsay and Jacoby (1994) sometimes said that $W$ and $C$ are measures of "influences" on responses, Lindsay and Jacoby might have had in mind that word reading and color naming jointly determine individual responses. However, interpreting $W$ and $C$ as degrees of influence renders their model uninterpretable. Without a processing model, we cannot determine how degrees of influence can be mapped to probabilities of responding. How much influence from a single process is needed in order for a response to be made? Will the same amount of influence accumulated from both processes together produce a response?

Furthermore, the equations are in terms of probabilities, not degrees. If $W$ is the probability that word reading influenced the correct response made, it is the probability of a binary occurrence, not of a graded occurrence. By having the probability of responding correctly on one side of the equation and the probabilities of influences on the other, there is an implied equivalence between responses and influences. Thus the equations only make sense when $W$ is interpreted as the probability that word reading determined the response made, where determined might be thought of as influenced beyond a response threshold. But this returns us to one of the first two interpretations of $W$ and $C$, in which there is no way to represent responses that were determined by word reading and color naming jointly.

The stochastic independence assumption seems plausible and coherent with interpreting $W$ and $C$ as influences: The degree to which (or the probability that) word reading influences the response is independent of the degree to which (or the probability that) color naming influences the response. But the dominance assumption has no justification: Why assume that a correct response cannot be made if word reading even partially influences the response?

Other interpretations. We have come up with three interpretations of $W$ and $C$, none of which seem compatible with both the process dissociation framework and the data it describes. It is possible, of course, that we have missed 
another interpretation. We therefore continue on to discuss other concerns with this dissociation of the Stroop task, in the hope that once an interpretation of $W$ and $C$ is found, the rest of our discussion will help in evaluating it. Because interpreting $W$ and $C$ as component reliabilities seems most consistent with the original intent of the article, we adopt that interpretation except where explicitly noted.

\section{Cognitive Architecture}

Stages and stochastic independence. Process dissociation focuses on color naming and word reading as holistic processes rather than as complex, multistaged processes. There is no doubt, though, that the processes are multistaged, consisting of at least perception of features, identification of features, and response selection, preparation, and execution. The extensive literature about decomposing processes into stages debates not the existence of stages but their organization (Donders, 1868/1969; Luce, 1986; McClelland, 1979; Miller, 1988; Pieters, 1983; Ratcliff, 1988; Schweikert, 1978; Sternberg, 1969; Taylor, 1976; Townsend \& Ashby, 1983).

Unfortunately, when color naming and word reading are addressed as multistaged processes, process dissociation's requisite assumption that the processes as a whole are stochastically independent seems unreasonable. And if that assumption is wrong, the estimates of $W$ and $C$ are wrong as well.

If two sequential processes share any stages, they are not stochastically independent. Consider, for instance, two processes, $M$ and $N$, that have stages, $x$ and $y$ respectively, that are independent of each other and independent of a shared stage, $z$. By definition, $M$ and $N$ are stochastically independent if the probability of both completing successfully is

$$
p(M \text { and } N)=p(M) p(N),
$$

where $p(i)$ is the probability that stage or process $i$ finishes successfully. The probability of a process finishing successfully is the probability that all stages of the process finish successfully. Because we have stated that the stages within each process are independent,

$$
p(M)=p(x \text { and } z)=p(x) p(z),
$$

and

$$
p(N)=p(y \text { and } z)=p(y) p(z) .
$$

The probability of two processes finishing successfully is the probability that all stages in the two processes finish successfully, so

$$
p(M \text { and } N)=p(x \text { and } y \text { and } z)=p(x) p(y) p(z) .
$$

If $M$ and $N$ are stochastically independent, then $p(M$ and $N)$ should equal $p(M) p(N)$. However, the quantities are not equal because:

$$
p(M) p(N)=(p(x) p(z))(p(y) p(z))=p(x) p(y) p(z)^{2}
$$

Unless $p(z)=1, p(M) p(N)<p(M$ and $N)$, and so $M$ and $N$ are not stochastically independent. ${ }^{3}$

In the Stroop task, response production is shared for the two processes, and some aspects of perception may be shared, too. ${ }^{4}$ Many models of the Stroop task include another common stage, one that selects from responses that have been generated from the two processes (e.g., Morton \& Chambers, 1973; Posner \& Snyder, 1975). This is the mechanism that enables color responses to occur most of the time even though word responses are available earliest. Such a stage for resolving response competition would clearly make $W$ and $C$ dependent.

This argument that a shared stage violates the independence assumption works most clearly when considering the interpretation of $W$ and $C$ as component reliabilities. The part of the process that produces the dominance of word processing over color naming must clearly consider outputs from both stages, and so must be a shared stage. When $W$ and $C$ are interpreted as source probabilities, the fact that $W$ and $C$ are probabilities of mutually exclusive alternatives makes them stochastically dependent to begin with. But if Equations 3 and 4 are replaced by Equations 5 and 6, then $W$ and $C$ can be dissociated without assuming they are stochastically independent. Finally, when $W$ and $C$ are interpreted as probabilities of influence, there should be a stage that combines the influence of word reading and the influence of color naming in order to produce a response. So once again, there would be a shared stage, and so stochastic dependence.

How important is this violation of stochastic independence? Unfortunately, without knowing to what degree processes are dependent, it is impossible to estimate the degree to which parameter estimates are off. But Curran and Hintzman (1995) showed that when a model assumes stochastic independence, even relatively minor violations of independence have serious consequences for process dissociation logic (see also Curran \& Hintzman, 1997; Jacoby et al., 1997). When they applied process dissociation to correlated processes, variables that produced real effects on one process produced artifactual effects on the other process, thereby producing inaccurate parameter estimates and artifactual evidence of functional dissociation.

An alternative to measuring the degree to which the current equations ( 3 and 4 ) are inaccurate is to come up with equations that more accurately reflect the model of processing. Equations 5 and 6 do so for the source probability interpretation of $W$ and $C$. The following equations do so for

\footnotetext{
${ }^{3}$ We made a simplifying assumption that the shared stage was stochastically independent of both private stages. If the shared stage and at least one private stage are dependent, then the two processes are dependent anyway: $p(x$ and $z)<>p(x) p(z)$, so $p(x$ and $y$ and $z)<>p(x) p(y) p(z)$. The only exception is if $p(y)$ compensates exactly for $p(x$ and $z)$, which would be an unlikely occurrence for independent stages.

${ }^{4}$ Although it might be argued that color and form perception are independent, Lindsay and Jacoby (1994) recommended the technique as a general approach to tasks in which two processes are in opposition. Independence of perceptual stages must be evaluated whenever the method is used.
} 
the component reliability interpretation:

$$
p(\text { correct } \mid \text { congruent })=[W+C(1-W)] S,
$$

and

$$
p(\text { correct } \mid \text { incongruent })=[C(1-W)] S,
$$

where $W$ is the probability of accurate word identification, $C$ is the probability of accurate color identification, and $S$ is the probability of accurate completion of all shared stages, including response selection and production. These equations assume that the shared stages are independent of word and color identification.

The two equations now have three unknowns, so unless virtually none of the errors are generated in the shared stages (i.e., unless $S$ is approximately 1), Equations 13 and 14 cannot be solved for $W$ and $C$. The shared responseresolution stage included by most models of the Stroop task would seem to be a potential source of nonnegligible errors. We have more to say about that later. The situation would be even less tractable if the shared stages were not independent of word and color identification. Without a better understanding of the nature of stages shared between word reading and color naming, it is not possible to determine whether Lindsay and Jacoby's (1994) data are interpretable.

Stochastic independence and functional independence.

Lindsay and Jacoby (1994) found that $W$ and $C$ are functionally independent. Color quality influences estimates of $C$ but not $W$, and the proportion of incongruent trials influences estimates of $W$ but not $C$. They interpreted these findings of functional independence as evidence of stochastic independence. It is possible, however, for two processes to be stochastically dependent and functionally independent, and vice versa (Tulving, 1984). Functional independence occurs when a variable that influences the probability of one thing occurring does not infuence the probability of another thing occurring. If two things, $M$ and $N$ are functionally independent, $p(M)$ can change without changing $p(N)$. Stochastic independence occurs when the probability of two things occurring together is equal to the product of the probabilities of each of those things occurring (see Equation 8). Whether $p(M) p(N)$ is equal to $p(M$ and $N)$ is not at all affected by what variables influence $p(M)$ and $p(N)$. The relationship expressed in Equation 8 could stay constant as $p(M)$ varied (i.e., if $p(M$ and $N)$ changed appropriately) or it could be violated as $p(M)$ changed (e.g., if $p(M$ and $N)$ was not influenced by the variable influencing $p(M)$ ). So there is no basis for expecting that two functionally independent processes will also be stochastically independent.

Consider the Stroop task. Color naming and word reading could plausibly be both functionally independent and stochastically dependent. We have already explained that if color naming and word processing share at least one stage, they would be stochastically dependent. And if color naming and word reading also have stages that are not shared, a variable could influence the private stages of one process while not influencing any other stages in either process. In this way color naming and word reading would be functionally independent while also being stochastically dependent. So
Lindsay and Jacoby (1994) have presented no evidence that color naming and word reading are stochastically independent.

\section{Imposing Deadlines: Taking the Time Out of Response Time}

Research on the Stroop task has predominantly measured speed of responding, for good reason. When people are confronted with the need to perform a less automatic cognitive task that is in competition with a more automatic cognitive task, their natural resolution is usually to slow their response rather than to do the wrong task. Thus, the predominant effect of congruency is in terms of speed, not errors. This is certainly true of the Stroop task.

Even so, the ability to account for both speed and accuracy has been a desideratum since at least the early 1960 s (for reviews of this early literature see, e.g., Luce, 1986; Townsend \& Ashby, 1983). The motivation for this is a general belief that accuracy cannot be understood without understanding time, and time cannot be understood without understanding accuracy. Accuracy develops over time, and theories must address that development (see, e.g., Meyer, Irwin, Osman, \& Kounios, 1988; Ratcliff, 1988). Theories that attempt to explain one without the other fall short of the prevailing ideal.

Models of the Stroop task have become more sophisticated over the years, and the best current models are approaching the ideal in that they account for both speed and accuracy and the relation between them (e.g., Cohen, Servan-Schreiber, \& McClelland, 1992; also see Cohen et al., 1990; Logan, 1980; Phaf et al., 1990). Furthermore, these models are now being challenged to account not only for response time and accuracy on individual trials, but also for entire distributions of response times (Heathcote, Popiel, \& Mewhort, 1991; Mewhort, Braun, \& Heathcote, 1992; see also Jacobs \& Grainger, 1994; Ratcliff \& Murdock, 1976, for the need to account for response time distributions in other tasks).

The process dissociation framework explains accuracy effects, but it does so at the expense of explaining response time effects. It cannot account for most of the response time results that have accumulated over the years. For instance, MacLeod (1991) listed 18 effects found in the response time literature that any successful model of the Stroop task must be able to account for. In fact, some existing models account for most of the effects (Cohen et al., 1990; Logan, 1980). The process dissociation framework has not been addressed toward most of the 18 effects, and without a great deal of elaboration, the framework would be unsuccessful in explaining them.

Furthermore, when a deadline is introduced in order to collect enough errors to measure accuracy effects, component processes may have been introduced into the architecture that were not important in unspeeded response time experiments. For instance, introducing deadlines is likely to increase guessing.

Response deadlines increase guessing. In the standard Stroop task, subjects usually respond with the color or the word and only rarely respond with a completely different 
color name, so guessing seems a negligible component of processing. In cognitive processing in general, subjects are likely to guess more often under fast deadlines than under slow (Ollman, 1966; Ollman \& Billington, 1972; Stanovich, 1979). So when Lindsay and Jacoby (1994) imposed a deadline, they may have increased the impact of guessing on responses.

The two-factor process dissociation model allows no independent role for bias or guessing. In order to include guessing as a factor within the framework provided by process dissociation, guessing must be inherent to either color naming or word reading rather than being independent of them. But guessing is often thought to be independent of both word reading and color naming or dependent on both jointly. For instance, when studying the effect of deadlines on response accuracy Ollman successfully modeled simple reaction data using a "fast guess" model that assumed that guessing (anticipating) was independent of principled processing (Ollman, 1966; Ollman \& Billington, 1972). The speed-accuracy decomposition technique reported by Meyer et al. (1988) provides a successful model of guessing driven by partial information from two sources.

If guessing is an influential independent process, the failure of process dissociation to embody it renders inaccurate the estimates of $W$ and $C$ from the current equations (Buchner, Erdfelder, \& Vaterrodt-Plünnecke, 1995; Roediger \& McDermott, 1994). (The data provided by Lindsay and Jacoby, 1994, do not allow us to determine the extent and basis of guessing. A record of how many responses matched neither the stimulus color nor the stimulus word would speak to the issue.) If guessing is influential and independent, Equations 3 and 4 could be adapted to reflect guessing (see Buchner et al., 1995, for an approach that could be adapted). The simplest adaptation would be based on an assumption that guessing occurs only when neither word reading nor color naming can control processing. This adaptation is made by simply adding the term $(1-W)$ $(1-C) G$ to the equations, which results in the following equations:

$$
\begin{aligned}
p(\text { correct } \mid \text { congruent })=W+ & (1-W) C \\
& +(1-W)(1-C) G,
\end{aligned}
$$

and

$$
\begin{aligned}
p(\text { correct } \mid \text { incongruent })=(1-W) C & \\
& +(1-W)(1-C) G,
\end{aligned}
$$

where $G$ is the probability of guessing correctly. Alternatively, if the decision to guess is the first decision made by subjects, as in the fast-guess model, then the equations might look like the following:

$$
\begin{aligned}
p(\text { correct } \mid \text { congruent })=G+ & (1-G) W \\
& +(1-G)(1-W) C,
\end{aligned}
$$

and

$$
p(\text { correct|incongruent })=G+(1-G)(1-W) C .
$$

Regardless of the way in which guessing is incorporated into the model, the two equations would now have three unknowns, which means the equations cannot be solved. 5

Post hoc deadlines. Deadlines in general introduce the concerns listed above but, in addition, we have concerns about the particular way in which post hoc deadlines were introduced by Lindsay and Jacoby (1994) to determine the time course of processing. Lindsay and Jacoby estimated $C$ and $W$ at a range of post hoc deadlines. In particular, they determined the probability of responding correctly by the deadlines and used those probabilities to generate $C$ and $W$. Note that the probabilities used were unconditional, $p$ (correct and by deadline), a method that treats nonresponses the same as error responses. ${ }^{6}$ This method may be problematic, for reasons we discuss later.

In contrast, consider two related probabilities. The first, the probability of responding by a certain deadline given that the response was correct, is the probability that makes up a cumulative distribution function (CDF; Townsend \& Ashby, 1983). It does not confound errors and nonresponses, but it is not useful for process dissociation because it is the probability of a response by a certain time (given that the response was correct), not the probability of a correct response.

The second related probability is the probability of responding correctly given that the response was made by a certain deadline. This conditional probability is a cumulative version of that used to study microtradeoffs of speed and accuracy, among other things (e.g., see Gratton, Coles, Sirevaag, Eriksen, \& Donchin, 1988; Lappin \& Disch, 1972). This type of probability could be used in process dissociation, because it is a probability of responding correctly.

Our concern is mostly that the probabilities used by Lindsay and Jacoby (1994) were unconventional. That does not mean, of course, that they were unacceptable. In order to make that judgment, one must understand what the probabilities were used for. What does it mean to determine the time

\footnotetext{
${ }^{5}$ Buchner et al. (1995) provided a formal treatment of how to adapt process dissociation to include the effects of guessing. The treatment assumes that the task being dissociated has only two response alternatives, so it cannot be applied directly to the Stroop task. But with modification, the treatment could probably apply.

${ }^{6}$ Note also that to look at the distribution of accuracy over time, vincentizing data is preferred over counting the number of responses made by post hoc deadlines. Binning data according to post hoc deadlines has the potential to give fast subjects more influence over early responses and slow subjects more influence over late responses. When that happens, the resulting distribution can be distorted such that it reflects no individual subjects' data. Vincentizing -ordering responses according to response time, dividing into bins containing equal numbers of responses, determining response time and accuracy for each subject within bin, and averaging these statistics across subject for each bin-tends to more accurately reflect the shape of individual subjects' distributions.
} 
course of $W$ and $C$ ? One possibility, that the time course represents growth of information about words and colors, can be ruled out: Information should be available before a response based on that information is made, so $W+C$ should always be larger than the probability of responding correctly. In contrast, because of the nature of Equations 3 and $4, W+C$ is always smaller than the probability of responding correctly (i.e., responding with the color name). ${ }^{7}$ To determine the growth of information, it would be more appropriate to apply different deadlines for different trials or blocks of trials and to estimate $C$ and $W$ on the basis of accuracies at these different a priori deadlines (see, e.g., speed-accuracy decomposition technique: Meyer et al., 1988, or response signal technique: McElree \& Dosher, 1989). Even so, for reasons we have discussed earlier, $W$ and $C$ are likely to reflect response resolution processes as well as word and color identification, so interpreting the time courses as merely information growth would be overly simplistic.

Another possibility is that the time course represents changes in the number of responses that were determined by word reading and the number of responses that were determined by color naming. If this is the intended meaning of a time course of $W$ and $C$, then the choice of whether to use conditional or unconditional probabilities to solve for $W$ and $C$ depends on how the time course is interpreted. Using conditional probabilities serves to compare $W$ and $C$ in terms of the influence they have had on responses that have already been made. Using unconditional probabilities compares $W$ and $C$ both in those terms and in terms of how they affect whether a response is made at all. Lindsay and Jacoby (1994) pointed out that word reading or color naming could occur without contributing to a response. They pointed out as well that $W$ and $C$ measure the probabilities that overt responses are infiuenced by word reading and color naming. So it seems odd to us to be looking at estimates of whether a response is made at all. For this reason, we would have preferred the use of conditional probabilities.

\section{Dominance}

Lindsay and Jacoby's (1994) characterization of processing assumes dominance of word reading over color naming. If $W$ and $C$ are interpreted as component reliabilities, this means that when both a word and a color response are possible, the word response always wins because word reading is automatic (see What Are $W$ and $C$ ?). Although subjects always intend to respond on the basis of the nonautomatic process, the automatic process is dominant and always overrides intention. ${ }^{8}$

The characterization of dominance is different from all previous and subsequent assumptions about dominance in the process dissociation literature. It has been customary to assume that the less automatic process dominates over the more automatic process (see, e.g., Jacoby, 1991; Yonelinas \& Jacoby, 1995). More important, Lindsay and Jacoby's (1994) characterization of dominance is contrary to both theoretical and empirical work on automaticity and the Stroop task. Automatic processes are difficult but not impossible to suppress (e.g., Logan, 1980, 1989; Shiffrin \& Schneider, 1977). And in countless experiments about the Stroop task (in which subjects were motivated to respond quickly but were not given a deadline), subjects made more errors when the word and color were incongruent than when congruent, but they easily obtained accuracies above $90 \%$. In other words, subjects are not compelled to respond with the word when they identify it. Color naming dominates word reading. Even when subjects have a relatively short time in which to respond, there is some evidence that they suppress already prepared word responses-they stumble over their vocalized responses, starting to say one word and then saying another. Thus, it appears that this characterization of the effect of automaticity is an outlier not only in terms of the field at large, but also in terms of work using the process dissociation method.

Granted, imposing a deadline could change the way response conflicts are resolved; subjects might respond incorrectly rather than more slowly, a situation that could be characterized by the model of dominance that Lindsay and Jacoby (1994) proposed. But Lindsay and Jacoby found the standard slowing of responses even when they imposed a deadline, and they found that responses were quite accurate, both of which suggest that deadlines did not change subjects' strategy to respond with the color rather than the word when they could.

It seems more appropriate, then, to assume that the intended process, color naming, dominates even though the automatic process, word reading, is more likely to finish by the deadline. This change would result in the following adjustments to base Equations 3 and 4:

$$
p(\text { correct } \mid \text { congruent })=C+(1-C) W,
$$

and

$$
p(\text { correct } \mid \text { incongruent })=C \text {. }
$$

Figure 2 shows estimates of $C$ and $W$ based on the data extracted from Lindsay and Jacoby's (1994) Figure 2. Panel A replicates part of their Figure 3, and Panel B shows the estimates when calculated using our new equations. Three changes can be noted. According to the new calculations, $W$ has more influence than $C$, the influence of $W$ increases or holds steady with time rather than decreasing, and both $C$ and $W$ are affected by changing color quality. We did not have the data set necessary for analysis of the significance of these apparent differences.

We have already stated that we think more adjustments need to be made to Equations 3 and 4 to account for the influence of shared stages and to account for guessing. So

\footnotetext{
${ }^{7}$ In Equation 3, $p$ (corr) $=W+C(1-W)=W+C-C W$, which is $C W$ less than $W+C$. In Equation $4, p$ (corr) $=$ $C(1-W)=C-C W$, which is $W+C W$ less than $W+C$.

${ }^{8}$ When $W$ and $C$ are interpreted as source probabilities, dominance is no longer an issue because response resolution is internal to the processing reflected by $W$ and $C$. When $W$ and $C$ are interpreted as influences, it is unclear how dominance would work.
} 


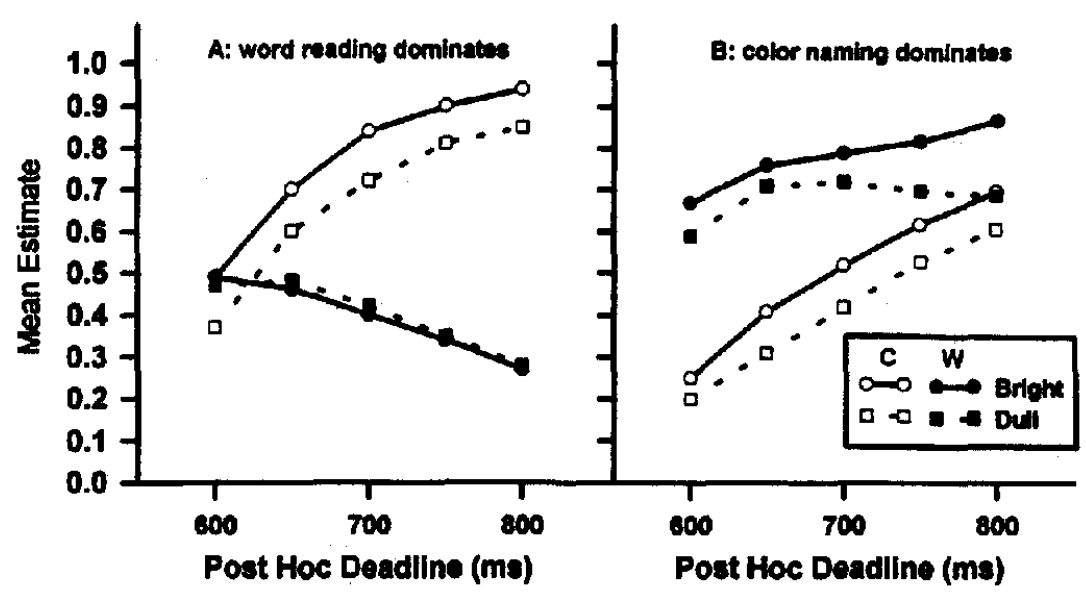

Figure 2. Estimates of $C$ and $W$ for data from Lindsay and Jacoby's (1994) Figure 2. Panel A shows their estimates, which assume word reading dominates over color naming. Panel B shows our estimates, which assume color naming dominates over word reading (see Equations 16 and 17).

we do not claim that the new data are veridical. But does one analysis give a better account of the Stroop task than the other? The results in Panel B seem as plausible to us as the results in Panel A. Both results show a strong influence of word reading early on, and a growing influence of color naming. The results in Panel B seem superior in that the infuence of word reading remains stronger than the influence of color naming over time. This is precisely the reason for assuming that color naming is given strategic dominance over word reading. Word reading, because it is the more automatic process, ought to influence processing earlier and more strongly than color naming, and its influence should not become weaker over time (e.g., Cattell, 1886; Lund, 1927). From this perspective, the results in Panel A are problematic because color naming somehow became stronger than word reading. However, the results in Panel A seem superior in that there seems to be no reason to expect color quality to impact word reading, and because Panel A's estimates of $C$ probably come closer to predicting performance in the neutral condition. ${ }^{9}$ This comparison of rather divergent results serves as a good demonstration that process dissociation is a method that needs a detailed model underpinning it, if its results are to be interpreted. The equations themselves represent a processing model that is not very detailed. Simply by changing one assumption in a reasonable way, two plausible patterns of results emerged that can only be evaluated in terms of more specific processing models.

As we mentioned, each dominance assumption results in estimates of $C$ and $W$ that would seem to support the opposite dominance assumption. When word reading is assumed to dominate, it is surprising that $W$ so seldom exceeds $C$. Likewise, when color naming is assumed to dominate over word reading, it is surprising that $C$ never exceeds $W$. What could it mean for word reading to dominate over color naming, if not that word reading more strongly influences responding than color naming?

Equation 20 may seem counterintuitive to some people because it asserts that correct responses to incongruent stimuli are not at all influenced by word reading. This is a reasonable criticism, but we do not believe the solution lies in accepting an unsupported assumption about dominance. Rather, we believe that it underlies the fact that the Stroop task cannot successfully be modeled by a two-process theory. We believe that trying to do so results not in errors that can be quantified by a "margin of error" estimate, but in qualitatively wrong results. Only when a shared response resolution process is added will the model have a chance of reflecting the effect of automaticity successfully.

\section{Summary of Concerns}

In summary, we believe problems exist with $W$ and $C$, with dependence between color naming and word reading, with the effects of imposing deadlines on the Stroop task, and with the incongruence between the automaticity of word reading and the assumed dominance of word reading over color naming. More articulated information could be collected to cast light on the nature of our concerns. We therefore ran the following experiment, replicating to a large degree the basic procedure used by Lindsay and Jacoby (1994).

\section{Experiment}

Subjects performed the Stroop task under three a priori deadline conditions (no deadline, $700 \mathrm{~ms}$ to respond, or 400

\footnotetext{
${ }^{9}$ Because we did not have data from individual subjects, we were not able to compare the two sets of equations in terms of the correlation between $C$ and performance in the neutral condition. The way in which our equations fit worse than Lindsay and Jacoby's (1994) equations would not be captured by that correlation, so the correlation might not have been much worse for our equations. On other measures of fit, our equations would have certainly fit worse than Lindsay and Jacoby's fit.
} 
ms to respond). We replicated several of Lindsay and Jacoby's (1994) analyses and, in addition, investigated many of our claims presented above. We tested whether imposing deadlines changed processing by seeing whether deadlines changed the probability of responding correctly and by seeing whether deadlines increased the amount of guessing. We explored how the data generated from post hoc deadlines differed when conditional rather than unconditional probabilities were used. We tested for a violation of independence by looking for evidence of guessing based on partial evidence about both word and color, and by looking for evidence of a common response resolution stage.

\section{Method}

Subjects. Twelve undergraduate students at the University of Illinois participated in the experiment for research credit for a psychology class. One subject demonstrated poor accuracy in the neutral condition, suggesting the possibility that he was colorblind, so his data were discarded. Two subjects informed the experimenter in the middle of the experiment that they had purposely averted their eyes from the stimulus in each display in order to perceive the color better than the word. Their data were discarded and subsequent subjects were instructed not to use that strategy. Three more undergraduates from the same subject pool were tested to replace the discarded data.

Apparatus. The stimuli were displayed on an AMDEK Model 722 color monitor driven by a VGA graphics card in an IBM AT computer. Subjects were seated so that their eyes were approximately $60 \mathrm{~cm}$ from the display. Vocal response times were measured using a Scientific Prototype Manufacturing Corporation, New York, Audio Threshold Detection Relay, Model 761G, which was driven by a Labtender card in the computer. The responses themselves were recorded by an experimenter sitting in the room with the subject. The experimenter faced away from the display except as noted in the procedure section. The data entry procedure allowed the experimenter to immediately correct any incorrect keystrokes she noticed, thereby minimizing response recording errors.

When the voice key did not register a vocalization, the experimenter requested the subject to repeat the response. (The voice key was insensitive to the experimenter's vocalizations.) Such responses were registered as unusually long-exceeding the longest post hoc deadline used in most of the data analyses.

Stimuli. On each trial, the subject first saw a fixation point at the center of the screen for $500 \mathrm{~ms}$ and then saw an upper-case letter string displayed in color against a black background until either a response was made or the deadline was reached, whichever came first. The letter string was approximately centered on the monitor screen: It was written beginning in column 38 of row 13 of a standard $80 \times 24 \mathrm{IBM}$ text screen. Letter strings were approximately $0.5^{\circ}$ of visual angle high and ranged from $0.9^{\circ}$ to $1.7^{\circ}$ wide. The set of letter strings used in the experiment were: $R E D, B L U E$, GREEN, WHITE, YELLOW, \%\%\%, \%\%\%\%, \%\%\%\%\%, and $\% \% \% \% \% \%$. The colors in which the letter strings appeared were red, blue, green, white, and yellow. Stimuli were either neutral (string of \%s written in a color), congruent (color name matching color in which it was written), or incongruent (color name different from color in which it was written). After the experimenter entered the response made, the screen was blank for $500 \mathrm{~ms}$ before the next stimulus appeared.

Procedure. Subjects were tested individually, and each subject completed the experiment in about 45-50 min. Before the experi- ment started, subjects were informed about the general task and were given 20 trials of practice with no response deadline. The experimenter observed the stimuli during practice to ensure that the subjects understood the task.

Practice was followed by three blocks of trials, with a short break in the middle of each and the opportunity for a long break between blocks. For one block, subjects had no response deadline; for another, subjects had $400 \mathrm{~ms}$ to respond, and for the third, subjects had $700 \mathrm{~ms}$ to respond. Two subjects were assigned to each possible ordering of blocks.

At the start of each block, the experimenter informed the subjects about the deadline condition for the block. For the $400 \mathrm{~ms}$ and $700 \mathrm{~ms}$ deadlines, subjects were told how long the deadline was and that they were to respond before the word disappeared from the screen. For the no deadline condition, subjects were told to respond quickly, but that there was no deadline.

Within each block, word and color were congruent in 80 trials and incongruent in 80 . The remaining 40 stimuli in each block were neutral. The stimuli within each congruency condition were divided equally among all possible word letter-string combinations for that condition.

The subjects were not told whether or not their responses were correct, but they were told when their responses did not meet the deadline. On such trials, the phrase TOO SLOW appeared in pink at the center of the screen for $1,000 \mathrm{~ms}$ after the experimenter entered the response and before the intertrial interval.

The deadlines we used are different from, but comparable to, the deadlines Lindsay and Jacoby (1994) used. Deadline was a within-subjects manipulation for us; it was a between-subjects (between-experiments, actually) manipulation for them. They used an $800 \mathrm{~ms}$ deadline in Experiment 2 and we used a $700 \mathrm{~ms}$ deadline. In their Experiments 3 and 4 , they gave an $800 \mathrm{~ms}$ deadline and required their experimenter to type the subject's response before the deadline. It is probably fair to estimate that their Experiments 3 and 4 deadlines were shorter than our $700 \mathrm{~ms}$ deadline and longer than our $400 \mathrm{~ms}$ deadline.

The remainder of our procedures are comparable to the procedures Lindsay and Jacoby (1994) used for the bright color condition in their Experiment 2. Notable differences are that they presented auditory feedback, whereas we presented visual feedback. They gave twice as many practice trials as we did, but we gave twice as many test trials as they did. And they used a longer interval between the experimenter's response and the appearance of the next stimulus $(2 \mathrm{~s})$ than we used $(0.5 \mathrm{~s})$.

\section{Results and Discussion}

Manipulation checks. Data that can be used to evaluate the effectiveness of the manipulations made are included in Table 1. All data are averaged across subjects. The percentage of particularly long responses is a conservative estimate of the number of voice-key errors: They occurred on fewer than $1 \%$ of trials. The percentages of trials beating the deadline indicate that subjects were not able to meet the 400 ms deadline reliably, but that they responded faster when they had a $400 \mathrm{~ms}$ deadline than when they had a $700 \mathrm{~ms}$ deadline. We were interested in relative rather than absolute deadlines, as were Lindsay and Jacoby (1994), so this manipulation was sufficient. And finally, the percentages of correct trials and the mean correct response times show that the Stroop effect obtained in both speed and accuracy measures. Thus, imposing deadlines was not completely effective in transferring the Stroop effect from response time 
Table 1

Percentage of Responses That Were Slow ( $>2 s$ ), That Beat Deadline, and That Were Correct, and Mean Response Times (in Milliseconds)

\begin{tabular}{|c|c|c|c|}
\hline \multirow[b]{2}{*}{ Response } & \multicolumn{3}{|c|}{ Deadline } \\
\hline & $400 \mathrm{~ms}$ & $700 \mathrm{~ms}$ & None \\
\hline \multicolumn{4}{|c|}{ Congruent stimuli } \\
\hline $\begin{array}{l}\text { \% slow } \\
\% \text { beat deadline } \\
\% \text { correct }^{\mathrm{a}} \\
\text { Response time }\end{array}$ & $\begin{array}{c}0.1 \\
33.7 \\
84.6 \\
443(82)\end{array}$ & $\begin{array}{c}0.0 \\
95.6 \\
98.1 \\
521(91)\end{array}$ & $\begin{array}{c}0.3 \\
\frac{-1}{99.0} \\
552(89)\end{array}$ \\
\hline \multicolumn{4}{|c|}{ Incongruent stimuli } \\
\hline $\begin{array}{l}\text { \% slow } \\
\% \text { beat deadline } \\
\% \text { correct } \\
\text { Response time }\end{array}$ & $\begin{array}{c}0.0 \\
26.0 \\
52.7 \\
556(120)\end{array}$ & $\begin{array}{c}0.2 \\
76.6 \\
80.8 \\
635(182)\end{array}$ & $\begin{array}{c}0.2 \\
\frac{-}{86.0} \\
664(121)\end{array}$ \\
\hline \multicolumn{4}{|c|}{ Neutral stimuli } \\
\hline $\begin{array}{l}\% \text { slow } \\
\% \text { beat deadline } \\
\% \text { correct } \\
\text { Response time }\end{array}$ & $\begin{array}{c}0.0 \\
23.1 \\
81.5 \\
492(70)\end{array}$ & $\begin{array}{c}0.0 \\
96.3 \\
96.7 \\
548(107)\end{array}$ & $\begin{array}{c}0.4 \\
-\overline{98.3} \\
578(74)\end{array}$ \\
\hline
\end{tabular}

Note. Standard deviations are in parentheses. Dashes represent not applicable.

apercentage of trials faster than $2,000 \mathrm{~ms}$ that were responded to correctly. 'Response times for trials with correct responses that were faster than $2,000 \mathrm{~ms}$.

to accuracy. Even under the $400 \mathrm{~ms}$ deadline, the mean response times were faster for congruent stimuli than for incongruent stimuli, $443 \mathrm{~ms}$ and $556 \mathrm{~ms}$ respectively, $t(11)=4.33, p<.01$.

Effect of deadlines. Subjects were only slightly less accurate when responding under the $700 \mathrm{~ms}$ deadline than when responding under no deadline. Responses under the $400 \mathrm{~ms}$ deadline were substantially less accurate. Imposing deadlines lowered accuracy of responding to all three kinds of stimuli: congruent, incongruent, and neutral. Responses to incongruent stimuli were most impacted.

We vincentized the data from the incongruent condition (Hockley, 1984). (The 80 trials for each subject were ordered according to RT, and binned into eight levels representing fastest to slowest responses, with 10 responses in each bin. Response time for each bin was determined by averaging response times within the bin for each subject and then averaging across subjects.) Figure 3 presents the proportion of word, color, and other responses within each bin, averaged across subjects, plotted against mean response time for each bin. Other responses were responses naming a color used in the experiment but not present (as either the color or word) in the current stimulus.

Comparing across a priori deadlines, one sees that when subjects had less time to respond (shorter a priori deadlines), they made more early responses. There were not only more fast word responses, there were also more fast color responses. But deadlines did not result in merely a sampling of faster responses from the same distribution. Responses of comparable speed were different under the different dead- lines. Thus, deadlines changed the nature of processing rather than simply transferring the Stroop effect from response time to accuracy.

Post hoc deadlines. The percentage of correct responses for the three a priori deadlines and post hoc deadlines ranging from $600 \mathrm{~ms}$ to $1,200 \mathrm{~ms}$ are presented in Figure 4, with unconditional probabilities (as calculated by Lindsay

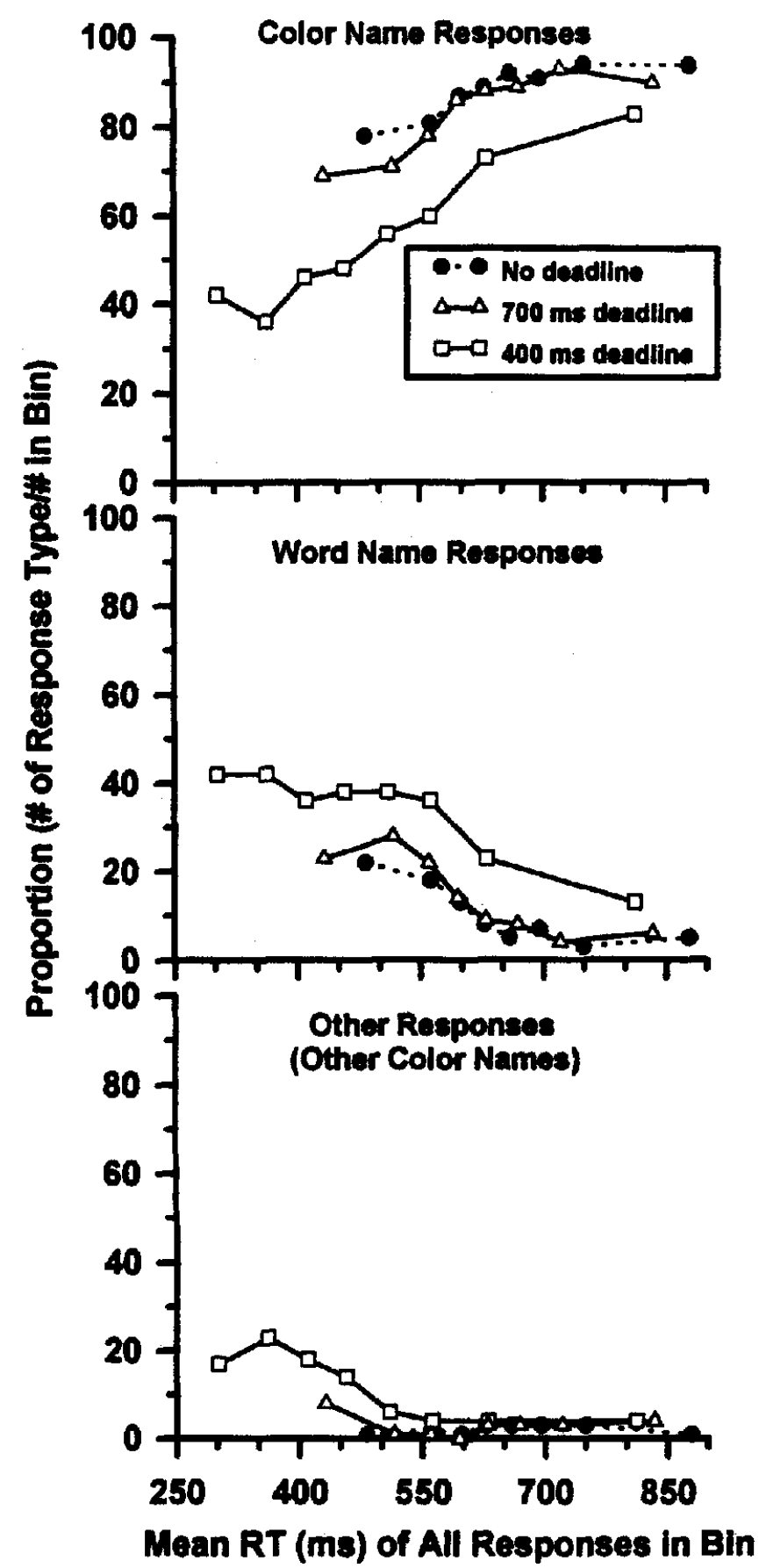

Figure 3. Average proportion of stimulus word, stimulus color, or other responses in each bin of vincentized data from the incongruent condition. 


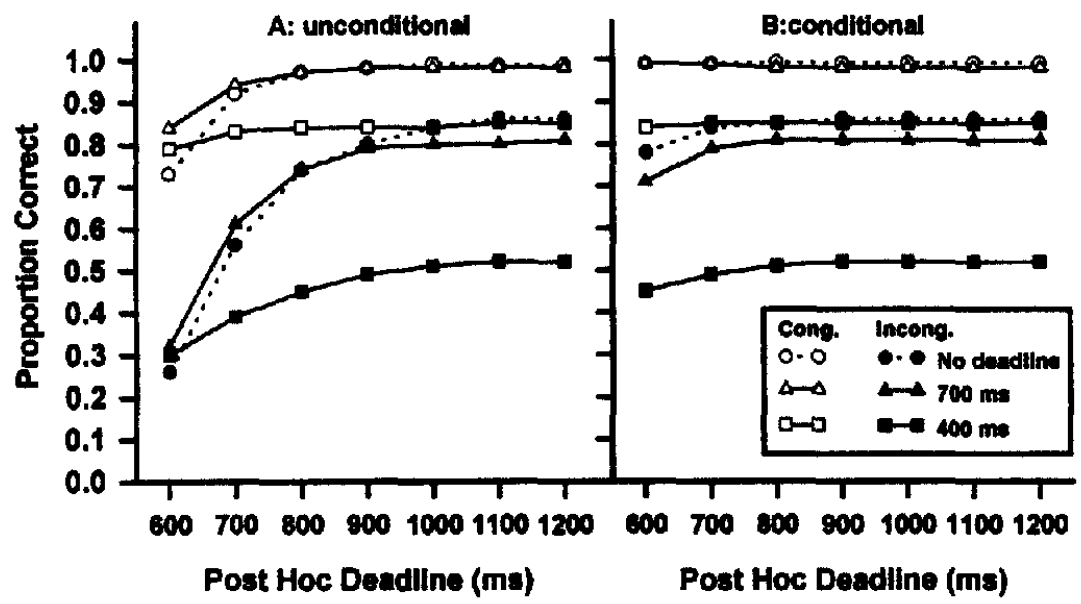

Figure 4. Percentage of correct responses for the three a priori deadlines and post hoc deadlines ranging from $600 \mathrm{~ms}$ to $1,200 \mathrm{~ms}$. Panel A shows unconditional probabilities (as calculated by Lindsay and Jacoby, 1994), and Panel B shows conditional probabilities (percentage of responses that were correct given that a response was made by the post hoc deadline).

and Jacoby, 1994) in Panel A and conditional probabilities in Panel B. Panel B highlights that under any particular a priori deadline, fast responses were just as accurate as slower responses, particularly in the congruent condition. This can also be seen for incongruent stimuli in Figure 3: There were as many word responses as color responses early on, but word responses were never substantially more likely, even for the fastest responses. The gradual rise in unconditional probabilities reported in Panel A and by Lindsay and Jacoby occurred not just because of fast errors, but because some correct responses took longer than others. And subjects were slower to respond in the incongruent conditions than in the congruent conditions.

Process dissociation was applied to the values in Figure 4, resulting in the estimates of $C$ and $W$ presented in Figure 5. Panel A is based on unconditional probabilities, calculating $C$ and $W$ using Lindsay and Jacoby's (1994) equations (Equations 3 and 4 in our article) to represent the dominance of word reading over color naming. Thus, this panel can be used to see whether our experiment replicated Lindsay and Jacoby's results. Our point of crossover of $C$ and $W$ was earlier than that found by Lindsay and Jacoby. In fact, the crossover point for the $400 \mathrm{~ms}$ a priori deadline happened earlier than $600 \mathrm{~ms}$, which was the earliest point at which all subjects responded in all conditions. The early crossovers are apparently due to the greater time pressure our subjects were under. Although there was no intended time pressure for the no deadline condition, there is reason to believe that subjects were pressuring themselves to respond faster in this experiment than in the average Stroop experiment without a deadline; in a pilot experiment providing no feedback about whether subjects met the deadline when one was given, response times in both deadline and no deadline conditions were over $100 \mathrm{~ms}$ slower than in this reported experiment.

Panel B of Figure 5 displays values of $C$ and $W$ calculated using Equations 3 and 4 and conditional probabilities. $C$ was larger than $W$ regardless of the type of probability (in both
Panel A and $B$ ), but the difference was greater when based on conditional probabilities (in Panel B). Changing from unconditional to conditional probabilities (comparing Panel A with Panel B) resulted in a different interpretation of the time course of the influence of color naming and word reading. When unconditional probabilities were used, the influences of the two processes changed over time; when conditional probabilities were used, the relative influences of the processes were stable over time.

Dominance. Panels C and D of Figure 5 display estimates of $C$ and $W$ based on unconditional and conditional probabilities, respectively, with $C$ and $W$ calculated using Equations 16 and 17 to represent the dominance of color naming over word reading. Assuming that color naming dominated resulted in a change in the estimated time course of the influence of word reading: The influence did not decrease over time. And in contrast to what was seen in Panels $\mathrm{A}$ and $\mathrm{B}, W$ was larger than $C$ in Panels $\mathrm{C}$ and $\mathrm{D}$. Changing from an assumption of word reading dominance to an assumption of color naming dominance (comparing Panel A with Panel C and Panel B with Panel D) resulted in a switch in the relative influence of the two processes. As before, when word reading was assumed to be dominant, color naming had a stronger influence than word reading. According to the component reliability interpretation of $C$ and $W, C$ could be larger than $W$ only if color naming finished successfully much more often than word reading. When color naming was assumed to be dominant, word reading had a stronger influence than color naming, suggesting that word reading finished successfully more often than color naming. We can think of no reason for these counterintuitive results.

To the degree that post hoc deadlines can be used to accurately estimate the time course of processing, Figures 4 and 5 demonstrate that imposing deadlines on subjects changes the time course fundamentally. If a priori deadlines had no effect on the processes underlying performance, then 

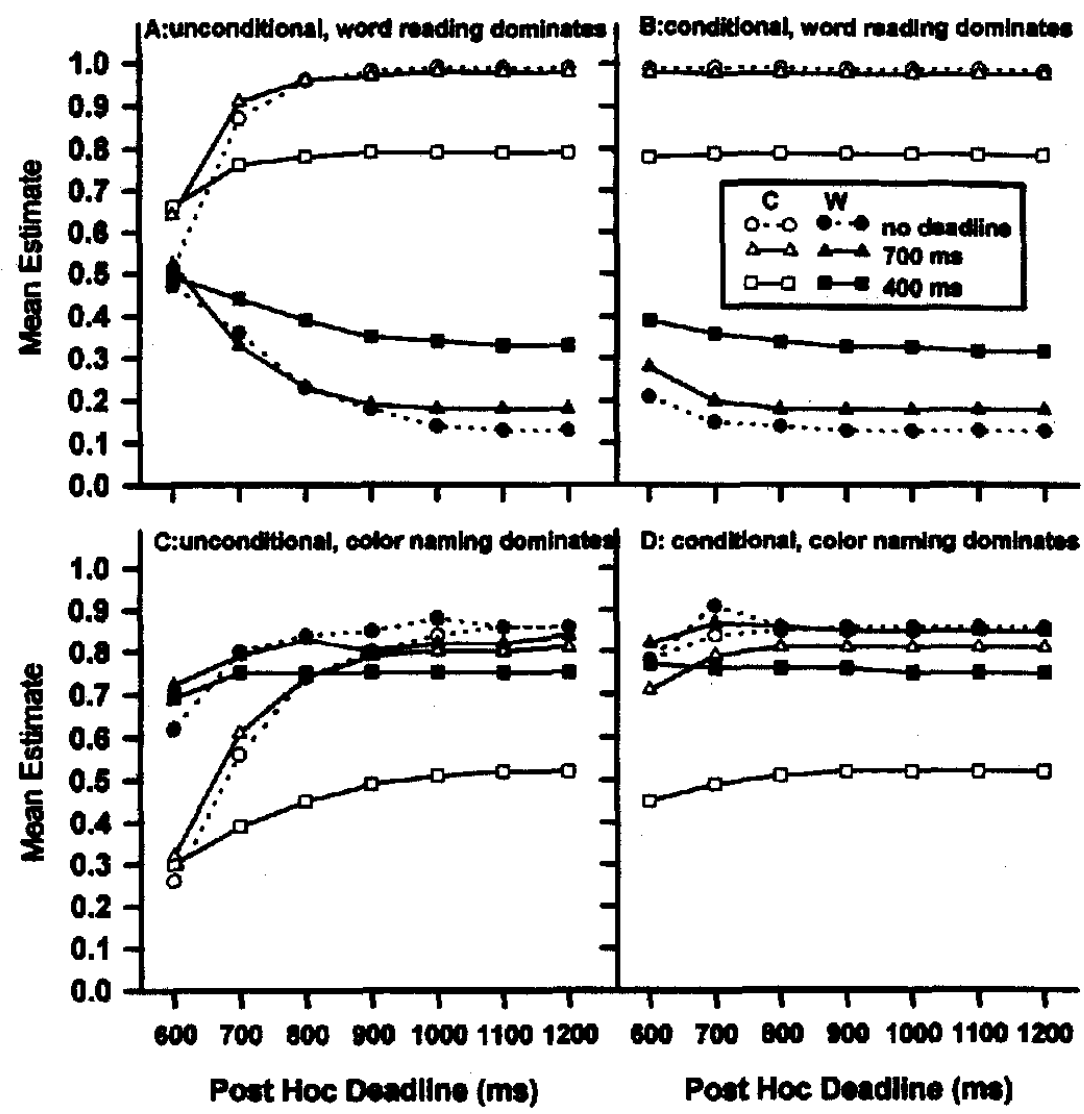

Figure 5. Estimates of $C$ and $W$ based on the percentage of correct responses displayed in Figure 3. Panels $A$ and $C$ are based on unconditional probabilities and Panels $B$ and $D$ are based on conditional probabilities. In Panels $A$ and B, $C$ and $W$ are calculated using Lindsay and Jacoby's (1994) equations (Equations 3 and 4). In Panels $C$ and D, $C$ and $W$ are calculated using Equations 16 and 17, which assume color naming dominates over word reading.

Table 2

Responses Matching Neither the Stimulus Color Nor the Stimulus Word: Frequency, Frequency Corrected to Be Estimate of Guessing, and Frequency as Percentage of All Responses

\begin{tabular}{|c|c|c|c|}
\hline \multirow[b]{2}{*}{ Frequency } & \multicolumn{3}{|c|}{ Deadline } \\
\hline & $400 \mathrm{~ms}$ & $700 \mathrm{~ms}$ & None \\
\hline \multicolumn{4}{|c|}{ Congruent stimuli } \\
\hline $\begin{array}{l}\text { Base } \\
\text { Corrected } \\
\text { As \% of responses }\end{array}$ & $\begin{array}{l}12.3 \\
15.4 \\
19.3\end{array}$ & $\begin{array}{l}1.3 \\
1.6 \\
2.0\end{array}$ & $\begin{array}{l}0.8 \\
1.0 \\
1.3\end{array}$ \\
\hline \multicolumn{4}{|c|}{ Incongruent stimuli } \\
\hline $\begin{array}{l}\text { Base } \\
\text { Corrected } \\
\text { As \% of responses }\end{array}$ & $\begin{array}{l}10.0 \\
16.7 \\
20.9\end{array}$ & $\begin{array}{l}2.8 \\
4.7 \\
5.9\end{array}$ & $\begin{array}{l}2.2 \\
3.7 \\
4.6\end{array}$ \\
\hline \multicolumn{4}{|c|}{ Neutral stimuli } \\
\hline $\begin{array}{l}\text { Base } \\
\text { Corrected } \\
\text { As \% of responses }\end{array}$ & $\begin{array}{r}7.3 \\
9.1 \\
22.8\end{array}$ & $\begin{array}{l}1.3 \\
1.6 \\
4.0\end{array}$ & $\begin{array}{l}0.6 \\
0.8 \\
2.0\end{array}$ \\
\hline
\end{tabular}

in Figure 4 the functions from the different deadline conditions should fall on top of each other, and in Figure 5 the a priori deadline conditions should not change estimates of $C$ and $W$. The clear separation of the functions in each figure suggests that the processing underlying performance was changed as deadlines were imposed. This can also be seen in Figure 3, which shows that deadlines did not result in merely a sampling of faster responses from the same distribution of responses. Responses of comparable speed were different in terms of accuracy under the different deadlines.

Guessing. To measure guessing, we counted responses that were in the set of colors used in the experiment but that matched neither the word nor the color of the stimulus. We corrected by a multiplicative factor of $5 / c$, where $c$ is the number of choices that were not the word or the color of the response -4 for the congruent and neutral conditions, 3 for the incongruent condition (see Table 2). ${ }^{10}$ If guessing was random (see, e.g., the fast-guess model; Ollman, 1966),

\footnotetext{
${ }^{10}$ Subjects never reported something other than the colors used in the experiment. There were a few trials (far fewer than $1 \%$ of trials) in which the experimenter coded no response at all.
} 
guessing would result in a response matching neither the word nor the color on $c$ out of 5 trials in which guessing was used. The correction factor, therefore, allows us to estimate the total amount of guessing based on the observable portion (if guessing is random). Note also that if guessing is random, subjects should be guessing on the same percentage of trials regardless of stimulus congruency.

Figure 3 shows more detailed data about guessing (uncorrected) for just the incongruent stimuli. Under the $400 \mathrm{~ms}$ deadline, almost $20 \%$ of fast responses to incongruent stimuli named a color not in the display. Similar responses were made on fewer than $10 \%$ of responses under the 700 $\mathrm{ms}$ deadline, under no deadline, and for slower responses under the $400 \mathrm{~ms}$ deadline. Imposing a stringent deadline increased the frequency of fast responses unrelated to the stimulus, which is consistent with an increased likelihood of fast guessing.

We analyzed guesses (measured as a percentage of all responses with response times less than 2,000 ms) using an analysis of variance (ANOVA) with two within-subjects factors, a priori deadline $(400 \mathrm{~ms}, 700 \mathrm{~ms}$, or none) and congruency (congruent, incongruent, or neutral). There were main effects of both congruency, $F(2,22)=4.0, p<.05$, and deadline, $F(2,22)=5.7, p<.05$. Guessing rates were $7.6 \%, 10.4 \%$, and $9.6 \%$ for congruent, incongruent, and neutral stimuli, respectively. Imposing a deadline increased subjects' likelihood of guessing. This demonstrates that processing under a deadline is not always comparable to processing without a deadline. The interaction between congruency and deadline was not significant, $F(4,44)=1.3$, $p>.05$.

The results of this ANOVA speak to the nature of guessing. The fact that our measure of guessing was affected by congruency suggests that stimulus information affects either the type of guesses made or the likelihood of guessing. Thus, subjects are not guessing randomly. Equations 15-18 were suggested as ways to incorporate random guessing into the process dissociation framework. The evidence from these analyses suggests that these equations will not completely capture the guessing process. In particular, if only one $G$ is used, the equations would violate invariance because congruency impacts guessing (see Meyer et al., 1988, for a method to decompose two types of guessing).

We noticed when we examined data from individual subjects that there was great variability in subjects' likelihood of guessing (ranging from $1.4 \%$ to $30.2 \%$ of trials, median $=3.8 \%$, averaged across deadlines and congruency conditions). It seemed plausible that those subjects who guessed frequently were engaging in fast guessing, whereas subjects who guessed infrequently were engaging in guessing based on partial information. To see if frequency of guessing affected type of guessing, another ANOVA was conducted in which a factor of guessing likelihood (infrequent vs. frequent) was added. Subjects with a belowmedian level of guessing were assigned to the infrequent guessing group, and the remaining subjects were assigned to the frequent guessing group. If frequency affected type of guessing as we suspected, an interaction between frequency of guessing and congruency ought to occur: Subjects who guessed frequently would not demonstrate a congruency effect, whereas subjects who guessed infrequently would. The results were that no interactions involving congruency were significant: Guessing Frequency $\times$ Congruency, $F(2$, 20) $<1$; Deadline $\times$ Congruency, $F(4,40)=1.3$; Guessing Frequency $\times$ Deadline $\times$ Congruency, $F(4,40)<1$. Congruency significantly affected guessing, $F(2,20)=3.7$, $p<.05$. There is no evidence that subjects who guessed frequently used an overall different guessing strategy than subjects who guessed infrequently. All subjects appeared to guess on the basis of partial information.

As in the original analysis, the ANOVA showed that subjects were more likely to guess under faster deadlines, $F(2,20)=9.9, p<.01$. The ANOVA picked up the division of subjects into frequent $(M=16.2 \%)$ and infrequent $(M=2.2 \%$ ) guessers. Frequency of guessing interacted with deadline, $F(2,20)=9.1, p<.01$ : For infrequent guessers, guessing rate was relatively homogeneous across deadlines $(2.0 \%, 2.7 \%$, and $2.0 \%$ errors under no deadline, the $400 \mathrm{~ms}$ deadline, and the $700 \mathrm{~ms}$ deadline, respectively), but for frequent guessers, it was particularly high under the $400 \mathrm{~ms}$ deadline $(3.2 \%, 39.2 \%$, and $6.2 \%$ errors under no deadline, the $400 \mathrm{~ms}$ deadline, and the $700-\mathrm{ms}$ deadline, respectively).

It seems evident in Figure 3 for incongruent stimuli that subjects are most likely to invoke a fast guessing strategy under the $400 \mathrm{~ms}$ deadline. As a final analysis testing whether fast guessing was ever used, the data from the $\mathbf{4 0 0}$ ms deadline were subjected to an ANOVA with two factors: guessing frequency and congruency. There were more guess responses under the $400 \mathrm{~ms}$ deadline for subjects who guessed frequently $(39.2 \%)$ than for subjects who guessed infrequently $(2.7 \%), F(1,10)=10.9, p<.01$. There was no evidence that congruency significantly influenced the likelihood of guessing for either frequent or infrequent guessers: congruency, $F(2,20)=1.0$; Guessing Frequency $\times$ Congruency, $F(2,20)<1$. For frequent guessers, guessing rates were $38.0 \%, 37.9 \%$, and $41.7 \%$ for congruent, incongruent, and neutral stimuli, respectively; for infrequent guessers, rates were $0.5 \%, 3.8 \%$, and $3.7 \%$, respectively. Thus, a fast guessing strategy under the $\mathbf{4 0 0} \mathrm{ms}$ deadline cannot be ruled out.

Because guessing was sensitive to stimulus congruency in our analyses, we ran one further ANOVA on responses matching neither the stimulus word nor stimulus color, this time uncorrected for random guessing. We included only data from the $700 \mathrm{~ms}$ deadline and no deadline conditions to see if these responses, when uncorrected, were still sensitive to stimulus congruency. Stimulus congruency significantly affected response rates: $1.4 \%, 3.1 \%$, and $2.4 \%$ for congruent, incongruent, and neutral stimuli, respectively, $F(2,20)=$ $4.8, p<.05$. Subjects assigned to the frequent guesser condition made more of these responses than subjects assigned to the infrequent guesser condition $(3.3 \%$ and $1.3 \%$, respectively), $F(1,10)=5.6, p<.05$. Deadline did not significantly affect responses, $F(1,10)=1.9, p>.05$, and there were no significant interactions: Guess Rate $X$ Deadline, $F(1,10)=1.9, p>.05$; Guess Rate $\times$ Congru- 
Table 3

Speed of Responses Matching the Stimulus Color, the Stimulus Word, or Neither (in ms)

\begin{tabular}{lccc}
\hline & \multicolumn{3}{c}{ Deadline } \\
\cline { 2 - 4 } Response & $400 \mathrm{~ms}$ & $700 \mathrm{~ms}$ & None \\
\hline \multicolumn{4}{c}{ Congruent stimuli } \\
Color & $443(91)$ & $521(82)$ & $552(82)$ \\
Other & $393(92)$ & $516(18)$ & $653(25)$ \\
\hline \multicolumn{4}{c}{ Incongruent stimuli } \\
Color & $556(182)$ & $635(121)$ & $664(120)$ \\
Word & $471(134)$ & $546(97)$ & $607(98)$ \\
Other ${ }^{2}$ & $415(108)$ & $529(47)$ & $578(57)$ \\
\hline \multicolumn{5}{c}{ Neutral stimuli } \\
Color & $492(107)$ & $548(74)$ & $578(70)$ \\
Other & $387(86)$ & $499(70)$ & $557(34)$ \\
\hline
\end{tabular}

Note. Standard deviations are in parentheses.

aSubject responded with a color used in experiment that matched neither the color nor word of the stimulus.

ency, $F(2,20)<1$; Deadline $\times$ Congruency, $F(2,20)<1$; and Guess Rate $\times$ Deadline $\times$ Congruency, $F(2,20)<1$.

Response times provide further information about guessing responses. Table 3 presents response times for the word, color, and guessing responses. For incongruent stimuli under all three deadlines, guessing responses are on average fastest, followed by word responses, and finally color responses (see also Figure 3). For congruent and neutral stimuli, guessing responses are almost always faster than color responses. Thus, response times, too, are consistent with our assumption that these responses are not based on complete processing of the stimuli.

In summary, when deadlines are imposed, a substantial number of responses match neither the color nor the word of the stimulus. We believe these responses evidence guessing. An analysis of the likelihood of these kinds of responses suggests that guessing is driven by some stimulus information-word-color congruency in particular. In order for Lindsay and Jacoby's (1994) model of processing to account for guessing, guessing must be inherent to either word reading or color naming, but not the relationship between them. The fact that stimulus congruency affects guessing suggests that not only is guessing not inherent to a single process, but that it violates the invariance assumption.

\section{Conclusions}

We have expounded several concerns about the way process dissociation logic has been applied to the Stroop task (Lindsay \& Jacoby, 1994). (a) The method relies on an assumption of independence that disregards the multistaged and interrelated cognitive architectures of color naming and word reading. Any stages of processing that are shared will lead to stochastic dependence. The experiment provided evidence that guessing is affected by stimulus congruency, and so must in some sense be shared. Imposing a stricter deadline not only increased fast errors but also increased fast correct responses, suggesting the possibility that some postidentification, preresponse stage that would usually be involved in processing is not involved when deadlines are imposed. (b) We provided evidence that processing changes when deadlines are imposed. The shape of the function relating response probability to response time changes as deadlines are imposed. Guessing increases as deadlines become more rigorous. The degree of guessing also changes as stimulus congruency changed, which argues that underlying processing is not invariant across deadlines or conditions. The fact that incongruent condition responses are slower than congruent condition responses even when there is a deadline is not reflected in the model of processing, and the use of post hoc deadlines deals inadequately with the slowing. The Stroop effect is largely a response time effect, and imposing a deadline does not convert it completely to an accuracy effect. (c) Automatic processing is assumed to be dominant over intentional processing in determining responses. But both theory about automatic processing and empirical response time data for the Stroop task argue that intentional processing dominates over automatic processing. Regardless of which process is assumed to dominate, resulting estimates of $W$ and $C$ seem to support the opposite assumption.

Some readers might think we are pointing out problems that merely render estimates of $C$ and $W$ imprecise. But the crux of our criticism is that we see process dissociation as a mathematical tool that needs to be laid on top of a fairly detailed theory of processing to have meaningful results. We see large inconsistencies between the level of modeling that the process dissociation equations represent and what is already known about the Stroop task. As a result, we believe that using process dissociation results not in estimates of $C$ and $W$ that are off by a measurable magnitude, but rather in completely uninterpretable results. This is most evident in our example of how estimates of $C$ and $W$ change when we make plausible changes to the assumptions about dominance and to the type of probabilities used to calculate $C$ and $W$. Doing so resulted in completely different results that cannot be dismissed just by looking at the pattern of results. Thus, we do not think the current process dissociation framework of assumptions about Stroop processing has heuristic value. Furthermore, we have doubts that the framework can be reworked to deal effectively with what is primarily a response time effect.

We have frequently been challenged to explain why the patterns of results found by Lindsay and Jacoby (1994) are so consistent and interpretable given that we think the method used is flawed. We have a number of responses to this challenge. First, we have shown that the results found are inconsistent with any interpretation of $W$ and $C$ that we could come up with. Second, we have shown that when the equations are adjusted to reflect what is known about the relationship between automatic and nonautomatic processes, the results are not consistent with the benchmark that Lindsay and Jacoby used to evaluate their results: The ability of the data to account for responses in the neutral condition. Third, we have shown that when we varied the assumption of dominance simultaneously with the method of calculating 
response probabilities under post hoc deadlines, we came up with four divergent, plausible interpretations of the results. Thus, the pattern of results is not as diagnostic as one might think. Fourth, response time models account at least as well for the results that process dissociation accounts for, and in addition account for many more results. (See MacLeod, 1991 , for a list of 18 phenomena that must be accounted for by a complete model of the Stroop effect.) So the consistency of process dissociation results is an insufficient reason for choosing to use process dissociation.

And finally, we recently became aware of an application of process dissociation to the Stroop task that resulted in estimates of $W$ and $C$ that were irreconcilable with other automaticity results. The Stroop task was dissociated under single-task and dual-task conditions (West, 1996). One of the characteristics of an automatic process is that it is little affected by a concurrent task (see, e.g., Bahrick \& Shelly, 1958; Brown \& Carr, 1989; Logan, 1979; Schneider \& Fisk, 1982; Strayer \& Kramer, 1990). To the degree that word reading is automatic, word reading should be unaffected by a concurrent task, whereas color naming ought to be affected because it is much less automatic. One of two results should obtain. If the division of attention does not affect the Stroop response resolution process, then estimates of $W$ should remain constant and estimates of $C$ should diminish under dual-task conditions. If the division of attention results in poorer Stroop response resolution, then estimates of $W$ should increase and estimates of $C$ should diminish under dual-task conditions. However, in West's experiment, estimates of both $W$ and $C$ decreased substantially under dual-task conditions.

In previous research using process dissociation to investigate the effect of dividing attention on memory, attention was manipulated during study, not during the recall phase (Jacoby et al., 1993; Jacoby, Woloshyn, \& Kelley, 1989). In those experiments, dividing attention affected estimates of the less automatic memory process, recollection, but not the more automatic memory process, familiarity. Thus, the results by West (1996) are not only inconsistent with automaticity research using methods other than process dissociation (Bahrick \& Shelly, 1958; Logan, 1979; Schneider \& Fisk, 1982; Strayer \& Kramer, 1990) but also with research using process dissociation (Jacoby et al., 1989; Jacoby et al., 1993).

Of course, word reading is not completely automatic, and dividing attention does affect intentional word processing (Becker, 1976; Carr, 1992; Herdman, 1992; Kellas, Ferraro, \& Simpson, 1988; Mullin \& Egeth, 1989; Posner, Sandson, Dhawan, \& Shulman, 1989). But word reading is an interfering contributor to Stroop responses, and so primarily the automatic parts of it are involved in the Stroop task. Furthermore, manipulating the proportion of trials that are congruent and incongruent affects the amount of interference words have. When a large proportion of trials are incongruent, there is less interference. This has been taken as evidence that attention is used to suppress word processing even further. According to this line of reasoning, when there is a large proportion of incongruent trials, the divided attention manipulation ought to increase estimates of $W$.
West (1996) both manipulated the proportion of trials that were congruent and divided attention. For subjects who saw incongruent stimuli on $80 \%$ of trials, $W$ was approximately 0.16 under the single-task condition and 0.08 under the dual-task condition. Thus, in West's experiment, the dualtask manipulation appeared to affect the automatic component of word reading.

A model needs to be evaluated not only in terms of whether the results it produces are internally consistent, but also in terms of whether the assumptions on which it is based are plausible. The best models provide ways to check assumptions that are independent of the results of the analysis. Consider, for example, the work by Meyer et al. (1988). They, like Lindsay and Jacoby (1994), were decomposing a task into underlying processes. They chose to do so by decomposing reaction time distributions into component distributions. They were able to check their assumptions about processing consisting of a race between independent components by checking whether hazard functions for responses were ordered as required if processing was a race (in particular, race models require that $h(x)$ for the winner must be greater than $h(x)$ for individual runners).

The process dissociation framework allows no such analysis-free check. Regardless, we believe we have shown that the assumptions of process dissociation about stochastic independence and dominance are implausible, when evaluated in terms of what is known about automaticity and what is known about the Stroop task.

Process dissociation logic has generated interest in part because the models applied to the original problems were elegant, requiring only two simple equations and two unknowns (Jacoby, 1991). Most of our concerns are with the oversimplifications required to use such an elegant model of processing. We highlighted factors not in the model that might contribute to processing in the Stroop task, and so should be included in the equations that model processing. The problem with having more variables is that more equations are needed to determine a unique solution, and each new equation in turn requires another experimental condition. It is encouraging to see a recent use of the overall logic that is not bound to two equations and two unknowns (Yonelinas \& Jacoby, 1995). But we believe the models must be made more complex to determine valid solutions to the questions being addressed.

In general, with each new common stage or independent process that influences accuracy, an additional experimental condition is required to find a solution through the process dissociation method. The added complexity may be worth the trouble when trying to separate the influence of implicit and explicit memory, which seldom if ever can be dissociated by task dissociations. But the Stroop task does not represent such a situation. Color naming and word reading have impacts that are easily separable through task dissociations; without invoking the complexity of a well-controlled process dissociation experiment. People usually study the Stroop task not in order to determine the independent influence of color naming and word reading, but rather to understand the resolution process that is clearly needed to decide between the conflicting influences of the color 
naming and word reading. Process dissociation does not illuminate that resolution process; on the contrary, it requires strong assumptions about how that resolution takes place.

Lindsay and Jacoby (1994) expressed enthusiasm over the possibility that the process dissociation method can replace standard methods that use response time measures to test models of processing. ${ }^{11}$ They pointed out that response time measures inadequately determine the relative strength of interference and facilitation in the Stroop task and other tasks when they measure such strength by comparing performance to a baseline (see Jonides \& Mack, 1984, for a similar point). We contend that the process dissociation method is subject to many problems itself. Rather than abandoning response time in favor of accuracy, the field may best be served by pursuing models that try to account for both speed and accuracy and the relationship between them (e.g., Cohen et al., 1990; Logan, 1980; Luce, 1986; Ratcliff, 1978; Townsend \& Ashby, 1983).

11 The process dissociation method differs from many current models of processing in that it does not provide any compression of the data. There is one parameter- $C$ or $W$-for each data point. This is not unlike Donders's (1868/1969) subtraction method for analyzing response times, which required $N+1$ tasks to estimate $N$ stage durations. Sternberg's (1969) additive factors model was a considerable improvement, reducing an $M \times N$ factorial design (with $M \times N$ conditions) to one or two theoretical entities (one stage if $M$ and $N$ interacted; two stages if they were additive). Modern models that deal with speed and accuracy and the relation between them compress the data even more. Ratcliff's (1978) model of memory retrieval accounts for accuracy and entire distributions of response times with only four parameters. In the domain of automaticity, Logan's (1992) instance theory accounts for the shape of the learning curve, the shape of the reaction time distribution, and changes in the shapes of both over practice with only three parameters. The models underlying the process dissociation method are not nearly so parsimonious.

\section{References}

Bahrick, H. P., \& Shelly, C. (1958). Time sharing as an index of automatization. Joumal of Experimental Psychology, 56, 288293.

Becker, C. A. (1976). Allocation of attention during visual word recognition. Journal of Experimental Psychology: Human Perception and Performance, 2, 556-566.

Brown, T. L., \& Carr, T. H. (1989). Automaticity in skill acquisition: Mechanisms for reducing interference in concurrent performance. Joumal of Experimental Psychology: Human Perception and Performance, 15, 686-700.

Brown, T. L., Roos-Gilbert, L., \& Carr, T. H. (1995). Automaticity and word perception: Evidence from Stroop and Stroop dilution effects. Journal of Experimental Psychology: Learning, Memory, and Cognition, 21, 1395-1411.

Buchner, A., Erdfelder, E., \& Vaterrodt-Pluinnecke, B. (1995). Toward unbiased measurement of conscious and unconscious memory processes within the process dissociation framework. Joumal of Experimental Psychology: General, 124, 137-160.

Carr, T. H. (1992). Automaticity and cognitive anatomy: Is word reading "automatic?" American Journal of Psychology, 105, 201-237.
Cattell, J. M. (1886). The time it takes to see and name objects. Mind, 11, 63-65.

Cohen, J. D., Dunbar, K., \& McClelland, J. L. (1990). On the control of automatic processes: A parallel distributed processing model of the Stroop effect. Psychological Review, 97, 332-361.

Cohen, J. D., Servan-Schreiber, D., \& McClelland, J. L. (1992). A parallel distributed processing approach to automaticity. American Journal of Psychology, 105, 239-269.

Curran, T., \& Hintzman, D. L. (1995). Violations of the independence assumption in process dissociation. Journal of Experimental Psychology: Learning, Memory, and Cognition, 21, 531-547.

Curran, T., \& Hintzman, D. L. (1997). Consequences and causes of correlations in process dissociation. Journal of Experimental Psychology: Learning, Memory, and Cognition, 23, 496-504.

Donders, F. C. (1969). On the speed of mental processes. In W. G. Koster (Ed. and Trans.), Attention and performance II (pp. 412-431). Amsterdam, The Netherlands: North-Holland. (Original work published in 1868)

Eriksen, C. W., \& Schultz, D. W. (1979). Information processing in visual search: A continuous flow conception and experimental results. Perception \& Psychophysics, 25, 249-263.

Gratton, G., Coles, M. H., Sirevaag, E. J., Eriksen, C. W., \& Donchin, E. (1988). Pre- and poststimulus activation of response channels: A psychophysiological analysis. Journal of Experimental Psychology: Human Perception and Performance, 14, 331344.

Heathcote, A., Popiel, S. J., \& Mewhort, D. J. K. (1991). Analysis of response time distributions: An example using the Stroop task. Psychological Bulletin, 109, 340-347.

Herdman, C. M. (1992). Attentional resource demands of visual word recognition in naming and lexical decisions. Joumal of Experimental Psychology: Human Penception and Performance, $18,460-470$.

Hockley, W. E. (1984). Analysis of response time distributions in the study of cognitive processes. Journal of Experimental Psychology: Leaming, Memory, and Cognition, 10, 598-615.

Jacobs, A. M., \& Grainger, J. (1994). Models of visual word recognition-Sampling the state of the art. Joumal of Experimental Psychology: Human Penception and Performance, 20, 13111334.

Jacoby, L. L. (1991). A process dissociation framework: Separating automatic from intentional uses of memory. Journal of Memory and Language, 30, 513-541.

Jacoby, L. L., Begg, I. M., \& Toth, J. P. (1997). In defense of functional independence: Violations of assumptions underlying the process-dissociation procedure? Joumal of Experimental Psychology: Learning, Memory, and Cognition, 23, 484-495.

Jacoby, L. L., Toth, J. P., \& Yonelinas, A. (1993). Separating conscious and unconscious influences of memory: Measuring recollection. Journal of Experimental Psychology: General, 122, 139-154.

Jacoby, L. L., Woloshyn, V., \& Kelley, C. M. (1989). Becoming famous without being recognized: Unconscious influences of memory produced by dividing attention. Journal of Experimental Psychology: General, 118, 115-125.

Jonides, J., \& Mack, R. (1984). On the cost and benefit of cost and benefit. Psychological Bulletin, 96, 29-44.

Joordens, S., \& Merikle, P. M. (1993). Independence or redundancy? Two models of conscious and unconscious influences. Joumal of Experimental Psychology: General, 122, 462-467.

Kahneman, D., \& Chajczyk, D. (1983). Tests of the automaticity of reading: Dilution of Stroop effects by color-irrelevant stimuli. Joumal of Experimental Psychology: Human Perception and Performance, 9, 497-509.

Kellas, G., Ferraro, F. R., \& Simpson, G. B. (1988). Lexical 
ambiguity and the timecourse of attentional allocation in word recognition. Journal of Experimental Psychology: Human Perception and Performance, 14, 601-609.

Lappin, J. S., \& Disch, K. (1972). I. Effects of stimulus probability on choice reaction time. Journal of Experimental Psychology, 92, 419-427.

Lindsay, D. S., \& Jacoby, L. L. (1994). Stroop process dissociations: The relationship between facilitation and interference. Journal of Experimental Psychology: Human Perception and Performance, 20, 219-234.

Logan, G. D. (1979). On the use of a concurrent memory load to measure attention and automaticity. Journal of Experimental Psychology: Human Perception and Performance, 5, 189-207.

Logan, G. D. (1980). Attention and automaticity in Stroop and priming tasks: Theory and data. Cognitive Psychology, 12, 523-553.

Logan, G. D. (1989). Automaticity and cognitive control. In J. S. Uleman \& J. A. Bargh (Eds.), Unintended thought (pp. 52-74). New York: Guilford.

Logan, G. D. (1992). Shapes of reaction time distributions and shapes of learning curves: A test of the instance theory of automaticity. Journal of Experimental Psychology: Learning, Memory, and Cognition, 18, 883-914.

Logan, G. D., \& Zbrodoff, N. J. (1979). When it helps to be misled: Facilitative effects of increasing the frequency of conflicting stimuli in a Stroop-like task. Memory \& Cognition, 3, 166-174.

Luce, R. D. (1986). Response times: Their role in inferring elementary mental organization. New York: Oxford University Press.

Lund, F. H. (1927). The role of practice in the speed of association. Journal of Experimental Psychology, 10, 424-433.

MacLeod, C. M. (1991). Half a century of research on the Stroop effect: An integrative review. Psychological Bulletin, 109, 163-203.

McClelland, J. L. (1979). On the time relations of mental processes: An examination of systems of processes in cascade. Psychological Review, 86, 287-330.

McElree, B., \& Dosher, B. A. (1989). Serial position and set size in short-term memory: The time course of recognition. Journal of Experimental Psychology: General, 4, 346-373.

Mewhort, D. J. K., Braun, J. G., \& Heathcote, A. (1992). Response time distributions and the Stroop task: A test of the Cohen, Dunbar, and McClelland (1990) model. Journal of Experimental Psychology: Human Perception and Performance, 18, 872-882.

Meyer, D. E., Irwin, D. E., Osman, A. M., \& Kounios, J. (1988). The dynamics of cognition and action: Mental processes inferred from speed-accuracy decomposition. Psychological Review, 95, 183-237.

Miller, J. (1988). Discrete and continuous models of human information processing: Theoretical distinctions and empirical results. Acta Psychologica, 67, 181-257.

Morton, J., \& Chambers, S. M. (1973). Selective attention to words and colours. Quarterly Journal of Experimental Psychology, 25, 387-397.

Mullin, P. A., \& Egeth, H. E. (1989). Capacity limitations in visual word recognition. Journal of Experimental Psychology: Human Perception and Performance, I5, 111-123.

Ollman, R. T. (1966). Fast guesses in choice reaction time. Psychonomic Science, 6, 155-156.

Ollman, R. T., \& Billington, M. J. (1972). The deadline model for simple reaction times. Cognitive Psychology, 3, 311-336.

Phaf, H., van der Heijden, A. H. C., \& Hudson, P. T. W. (1990). SLAM: A connectionist model for attention in visual selection tasks. Cognitive Psychology, 22, 273-341.
Pieters, J. P. (1983). Sternberg's additive factor method and underlying psychological processes: Some theoretical considerations. Psychological Bulletin, 93, 411-426.

Posner, M. I., Sandson, J., Dhawan, M., \& Shulman, G. L. (1989). Is word recognition automatic? $\mathrm{A}$ cognitive-anatomical approach. Journal of Cognitive Neuroscience, 1, 50-60.

Posner, M. I., \& Snyder, C. R. R. (1975). Attention and cognitive control. In R. L. Solso (Ed.), Information processing and cognition: The Loyola symposium (pp. 55-85). Hillsdale, NJ: Eribaum.

Ratcliff, R. (1978). A theory of memory retrieval. Psychological Review, 85, 59-108.

Ratcliff, R. (1988). Continuous versus discrete information processing: Modeling accumulation of partial information. Psychological Review, 95, 238-254.

Ratcliff, R., \& Murdock, B. B. (1976). Retrieval processes in recognition memory. Psychological Review, 83, 190-214.

Roediger, H. L., III, \& McDermott, K. B. (1994). The problem of differing false alarm rates for the process dissociation procedure: Comments on Verfaellie and Treadwell (1993). Neuropsychology, 8, 284-288.

Schneider, W., \& Fisk, A. D. (1982). Degree of consistent training: Improvements in search performance and automatic process development. Perception \& Psychophysics, 31, 160-168.

Schweikert, R. (1978). A critical path generalization of the additive factors method: Analysis of a Stroop task. Journal of Mathematical Psychology, 18, 105-139.

Shiffrin, R. M., \& Schneider, W. (1977). Controlled and automatic human information processing: II. Perceptual learning, automatic attending, and a general theory. Psychological Review, 84, $127-190$.

Stanovich, K. E. (1979). Studies of letter identification using qualitative error analysis: Effects of speed stress, tachistoscopic presentation, and word context. Journal of Experimental Psychology: Human Perception and Peformance, 5, 713-733.

Sternberg, S. (1969). The discovery of processing stages: Extensions of Donders' method. In W. G. Koster (Ed.), Attention and performance $I I$ (pp. 276-315). Amsterdam, The Netherlands: North-Holland.

Strayer, D. L., \& Kramer, A. F. (1990). Attentional requirements of automatic and controlled processing. Journal of Experimental Psychology: Learning, Memory, and Cognition, 16, 67-82.

Stroop, J. R. (1935). Studies of interference in serial verbal reactions. Journal of Experimental Psychology, 18, 643-662.

Taylor, D. A. (1976). Stage analysis of reaction time. Psychological Bulletin, 83, 161-191.

Townsend, J. T., \& Ashby, F. G. (1983). Stochastic modeling of elementary psychological processes. Cambridge, England: Cambridge University Press.

Tulving, E. (1984). How many memory systems are there? American Psychologist, 40, 385-398.

West, R. L. (1996). The role and nature of attention in the Stroop task. Paper presented at the 68th Annual Meeting of the Midwestern Psychological Association, Chicago, IL.

Yonelinas, A. P., \& Jacoby, L. L. (1995). Dissociating automatic and controlled processes in a memory-search task: Beyond implicit memory. Psychological Research, 57, 156-165.

Received August 24, 1995

Revision received May 20, 1996 Accepted July 16, 1996 